\title{
IN-LINE DETERMINATION OF METABOLITES AND MILK COMPONENTS WITH ELECTROCHEMICAL BIOSENSORS
}

\author{
M. MASCINI* \\ Institute of Analytical Chemistry, University of Florence, Via G. Capponi 9, Florence (Italy) \\ D. MOSCONE, G. PALLESCHI and R. PILLOTON
}

Department of Science and Chemical Technology, II University of Rome, Via Orazio Raimondo, 00173 Rome (Italy)

(Received 24th March 1988)

\section{SUMMARY}

Electrochemical biosensors for lactate, pyruvate and $\beta$-hydroxybutyrate based on oxygen, hydrogen peroxide, and NADH sensors coupled with oxidase and dehydrogenase enzymes were developed and used in conjunction with an artificial pancreas in experiments with extracorporeal circulation. Such procedures allow the fate of these species involved in glucose metabolism to be clarified during insulin treatment of diabetic patients. Studies with a glucose oxidase electrode for in-line determination of glucose produced by hydrolysis of cellobiose in a bioreactor are reported; for the determination of glucose in the presence of high concentrations of cellobiose, the purity of glucose oxidase is important in obtaining linear calibration plots. Impurities like amylase, maltase, invertase, and galactose oxidase, which are usually present in commercial preparations of glucose oxidase, must be absent. Another application is the amperometric determination of lactose, lactate and glucose in milk samples by using a hydrogen peroxide sensor coupled with $\beta$-galactosidase, lactate oxidase and glucose oxidase. The procedures outlined are simple and the short response times enable milk to be monitored during processing.

Biosensors offer new possibilities for real-time monitoring of metabolites of medical, pharmaceutical or biotechnological interest. Examples of such possibilities are shown and evaluated in this paper, but first some general problems in clinical and industrial applications and in food technology that can be solved with electrochemical biosensors are outlined.

An example from clinical work is the recently developed artificial pancreas, Betalike (Esacontrol, Genova, Italy), which is a glucose-controlled insulin infusion system for diabetic patients. Blood, continuously pumped from the patient $\left(6 \mathrm{ml} \mathrm{h}^{-1}\right)$, is diluted $(1+9)$ with a physiological saline solution and passes through an ultrafiltration unit from which the particulate matter is reinfused back to the blood stream while the ultrafiltrate is analyzed for glucose. This glucose value activates the insulin delivery system at a rate controlled by a suitably programmed computer [1]. However, the infusion of insulin provokes certain changes in the concentrations of various metabolites, including 
L-lactate, pyruvate, alanine, and ketone bodies [2,3]. Information on the concentrations of these metabolites would be useful for establishing metabolic patterns in diabetic patients and for deriving a more precise algorithm for the insulin infusion $[4,5]$.

Biotechnology is changing industrial processes in several fields for the production of fine chemicals starting from waste materials. Cellulose is the most abundant and renewable raw organic material on this planet. Often considered as waste material, it can be exploited to produce fine chemicals by suitable transformation. The enzymatic hydrolysis of cellulose is of interest because it requires low energy consumption and produces compounds for the food and pharmaceutical industries. The hydrolysis of cellulose occurs in several steps involving several enzymes. $\beta$-Glucosidase (EC.3.2.1.20) is the main limiting factor in the enzymatic hydrolysis [6]. The use of whole cells with $\beta$-glucosidase activity, instead of the purified enzyme, in a hollow-fiber membrane reactor has been discussed [7]. However, the development of suitable bioprocesses needs fine tuning of several parameters $[8,9]$. The glucose concentration is an important value for controlling the bioreactor performance on line and in real time.

Another application of electrochemical biosensors is the determination of lactose, lactate and glucose in milk samples. Aerobic degradation of lactose produces lactic acid, thus the $\mathrm{pH}$, lactose [10] and lactate concentrations should be checked to establish the suitability of milk samples for human consumption. The normal lactate concentration in fresh milk samples is $1-2 \mathrm{mmol} \mathrm{l}^{-1}$ but it can be increased to $10-20 \mathrm{mmol}^{-1}$ by microbial fermentation. Lactose, which is usually present at concentrations of about $5 \%(\mathrm{w} / \mathrm{v})$ in milk samples, can be determined by using immobilized enzymes based on the following reactions: $\beta$-galactosidase cleaves the disaccharide, producing glucose and galactose; glucose is then oxidized in the presence of glucose oxidase to form hydrogen peroxide, which is measured with a platinum electrode polarized at $650 \mathrm{mV}$ vs. $\mathrm{Ag} / \mathrm{AgCl}$. In a previous paper [10], a sensor was described for the determination of lactose in various milk samples; the results correlated well with those obtained by a titrimetric method. Here, an improvement of the sensor suitable for use in a flow system is discussed. The determination of glucose is useful in processing milk to convert lactose to glucose. These processes produce milk more suitable for consumption by people with genetic diseases in the synthesis of $\beta$-galactosidase, so that precise and careful quality control is necessary.

\section{EXPERIMENTAL}

\section{Materials}

L-Lactate oxidase (EC.1.2.3.2; activity $18 \mathrm{U} \mathrm{mg}^{-1}$ ) and pyruvate oxidase (EC.1.2.3.3; $21 \mathrm{U} \mathrm{mg}^{-1}$ ) both from Pediococcus sp. were obtained from Toyo Jozo Co. (Shizuoka, Japan). $\beta$-hydroxybutyrate dehydrogenase (EC.1.1.1.30 
from Rhodopseudomonas Spheroides Type II), $\beta$-galactosidase (EC.3.2.1.23, from Escherichia Coli; $580 \mathrm{U} \mathrm{mg}^{-1}$ solid) and glucose oxidase (EC.1.1.3.4, from Aspergillus Niger, type V; $1200 \mathrm{U} \mathrm{ml}^{-1}$ ) were obtained from Sigma. All other reagents and buffers were of analytical-reagent grade.

Poly (azetidine) prepolymer solution (Hercules Polycup 172, $12 \%$ solids in water) was obtained from M. Delaney (New York, 10010). Cellulose acetate (53\% acetyl) and poly (vinyl acetate) with high molecular weight were obtained from Farmitalia Carlo Erba. The 120-mesh nylon net used (A. Bozzone, Appiano Gentile, Italy) was $100-\mu \mathrm{m}$ thick, with $35 \%$ of its area as free surface. The Biodyne immunoaffinity membrane, nylon $66(0.2-\mu \mathrm{m}$ pore size $)$ with carboxylic groups on the surface was obtained from Pall Filtration Corporation (Glen Cove, NY). The dialysis membrane ( 0.001 in.-thick with molecular cutoff 12000 ) was obtained from A. H. Thomas Co. (Philadelphia, PA).

For casting the cellulose membrane, the precision gauge tool used was obtained from Precision Gauge and Tool Co. (Dayton, $\mathrm{OH}$ ). A reactor provided with polysulfonic hollow fibers (molecular weight cutoff 40000 ) was kindly supplied by Esacontrol (Genova, Italy). The hydrogen peroxide sensor was obtained from Instrumentation Laboratory. Platinum wire was $1 \mathrm{~mm}$ in diameter; the area of the silver foil cathode was $0.5 \mathrm{~cm}^{2}$. The polarization unit was a VA detector, Metrohm 641 voltammeter and the current was displayed on an Omniscribe recorder. The Betalike artificial pancreas is produced by Esacontrol.

\section{General procedures}

Immobilization of enzymes. Several immobilization techniques were tested for each enzyme. The final choice was based on the residual activity after treatment, the response and recovery times and the lifetime. L-Lactate oxidase was immobilized on nylon net as previously described [11]. Pyruvate oxidase was immobilized by chemical bonding on a Pall Biodyne membrane [12]. $\beta$ - $\mathrm{Hy}-$ droxybutyrate dehydrogenase was immobilized by physical adsorption on a pretreated graphite electrode. $\beta$-Galactosidase was immobilized by the polyazetidine procedure [13]. Glucose oxidase was immobilized by the bovine serum albumin procedure.

Constructon of the sensors. All sensors used in this work except for the $\beta$ hydroxybutyrate probe were assembled in the following way. A membrane of cellulose acetate was placed on the platinum surface to eliminate interferences from electroactive compounds such as ascorbic acid. A second membrane with immobilized enzyme was placed on top of the first one and covered with a dialysis membrane in order to prevent microbial attack and leaching of the enzyme from the second membrane. The three layers were kept in position with a rubber $\mathrm{O}$-ring. The sensors were fitted in a flow cell through which standard and sample solutions were aspirated by a peristaltic pump, or they were used in a beaker. 
Construction of the $\beta$-galactosidase reactor. The $\beta$-galactosidase reactor was based on polysulfonic hollow fibers coated with the immobilized enzyme. The coating solution contained $\beta$-galactosidase $(1 \mathrm{mg})$ and polyazetidine $(15 \mu \mathrm{l}$ of a $12.5 \%$ aqueous solution) in $150 \mu \mathrm{l}$ of phosphate buffer (pH 7.0, $0.1 \mathrm{~mol} \mathrm{l}^{-1}$ ). This solution was pumped, with a peristaltic pump, into the reactor at a flow rate of $0.1 \mathrm{ml} \mathrm{min}^{-1}$.

\section{RESULTS AND DISCUSSION}

\section{Experiments with the Betalike artificial pancreas}

A flow cell containing the lactate and pyruvate sensors was placed downstream from the glucose sensor of the Betalike artificial pancreas apparatus. The flow rate of the dialysate was fixed at $0.2 \mathrm{ml} \mathrm{min}^{-1}$. Through a T-connection, a peristaltic pump introduced a concentrated solution of cofactors into the flowing dialysate, at a flow rate of $0.02 \mathrm{ml} \mathrm{min}^{-1}$, to provide the optimum cofactor concentrations in the solution entering the measuring flow cell.

Figure 1 shows the results of an in vivo determination of glucose, L-lactate, and pyruvate in heparinized blood from a normal subject (a 23 year-old male nondiabetic volunteer) being treated with the artificial pancreas (Betalike). Blood taken from the patient via a double-lumen catheter $\left(6 \mathrm{ml} \mathrm{h}^{-1}\right)$ was heparinized, diluted 10 -fold with physiological saline buffer and then dialyzed through the dialysis filter. The dialysate was pumped $\left(0.2 \mathrm{ml} \mathrm{min}^{-1}\right)$ through the glucose sensor and then through the flow cell containing the lactate and pyruvate sensors. Every 15-20 min, blood samples were collected from the patient and lactate and pyruvate were determined spectrophotometrically within the next few days. These results, represented as circles in Fig. 1, illustrate the agreement of the results obtained by both methods. At the time marked ST, a standard solution of lactate and pyruvate was passed through the cell to calibrate the sensors. At the time marked CAL, the glucose sensor of Betalike was calibrated; the blood flow was disconnected from the sensors during such periods. At the time marked EX, the patient was requested to do a short physical exercise, which was stopped at the time marked STOPEX. At the time GLU INF, a dose of glucose $(50 \mathrm{~g})$ was infused within a short period of time. MEAL, indicates the time at which the patient ate a normal meal. It is interesting to note the large effect of physical exercise on the concentration of lactate and, even more, pyruvate, followed by the parallel return of both species to "normal" concentrations. As expected, the glucose dosage had a large effect on glucose concentration, but caused only small variations in the concentrations of lactate and pyruvate. After the meal, all three analytes showed large and continuous variations.

Numerous factors influence the consumption of compounds; each factor can produce different variations in metabolic substrates. The lack of insulin that characterizes diabetes causes these variations to assume pathological values. 


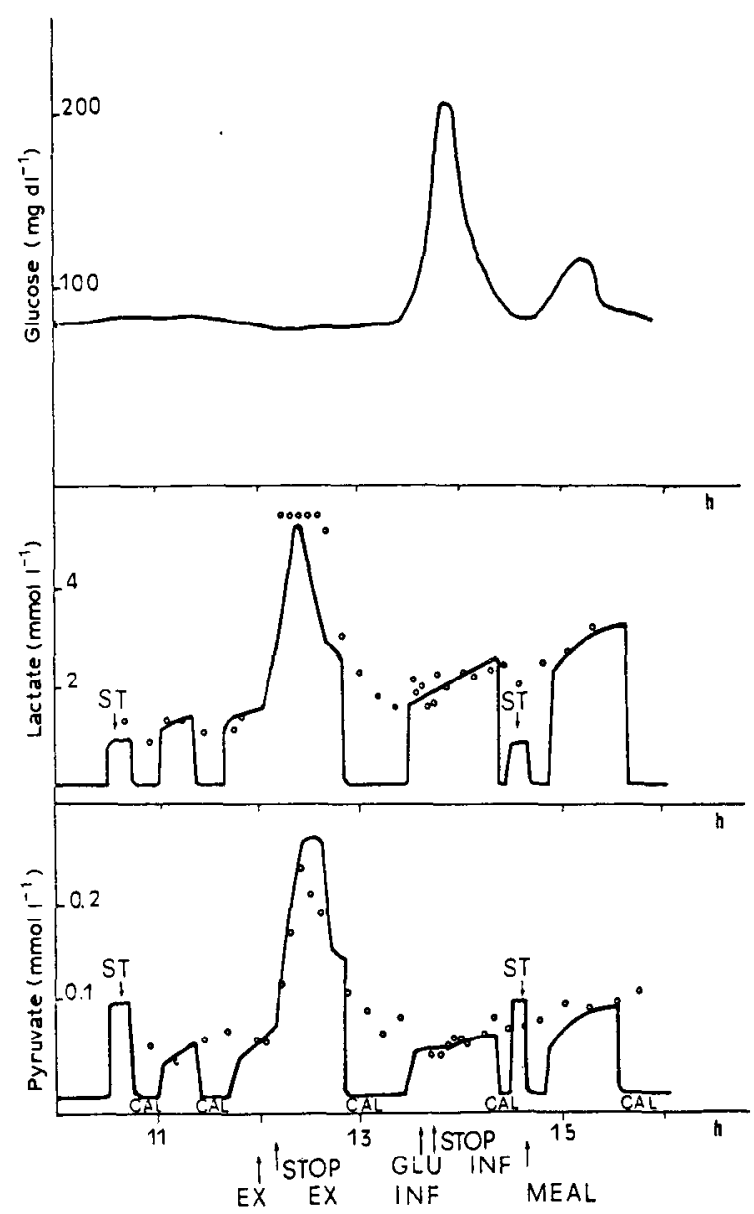

Fig. 1. Continuous monitoring of glucose, lactate and pyruvate in vivo during an experiment with the artificial pancreas "Betalike". See text for explanations of abbreviations.

The correction of these variations, to complete normalization, will be possible only if the insulin replacement therapy is regulated by taking into account the complex relationships between the various intermediate metabolites [2,3]. The possibility of creating algorithms that allow optimal therapeutic results is strictly governed by the amount of information available. The immobilization procedures described here provide the basis for reliable sensors that can be used for such studies of regulating the insulin therapy of diabetes.

For the determination of $\beta$-hydroxybutyrate, preliminary tests were done by measuring this metabolite at concentrations corresponding to the level in 10 fold diluted blood. Because the enzymatic reaction involves the $\mathrm{NAD}^{+}$cofactor, a graphite electrode was selected as NADH sensor and the current arising from the electrochemical oxidation of $\mathrm{NADH}$ produced by the enzymatic re- 


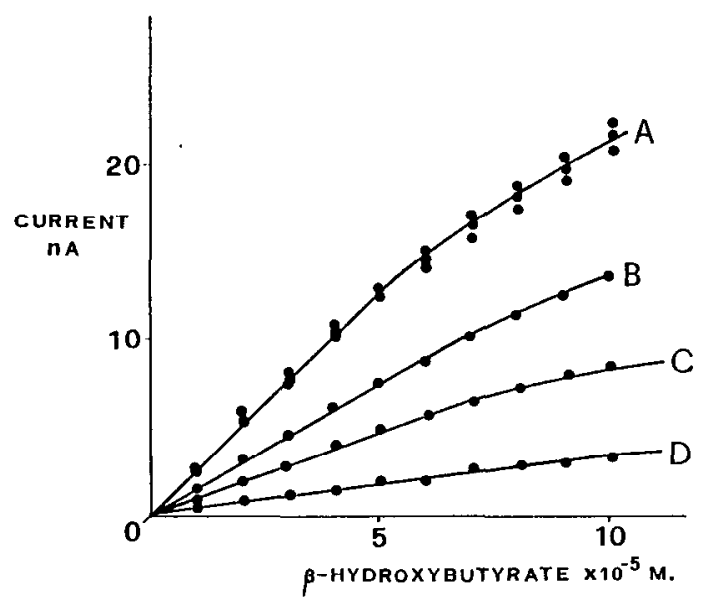

Fig. 2. Calibration cures for $\beta$-hydroxybutyrate at different times. Phosphate buffer, $\mathrm{pH} 7.5, \mathrm{NAD}^{+}$ $0.5 \mathrm{mmol} \mathrm{l}^{-1} ; 25^{\circ} \mathrm{C}$. (A) First day; (B) after 4 days; (C) after 7 days; (D) after 10 days.

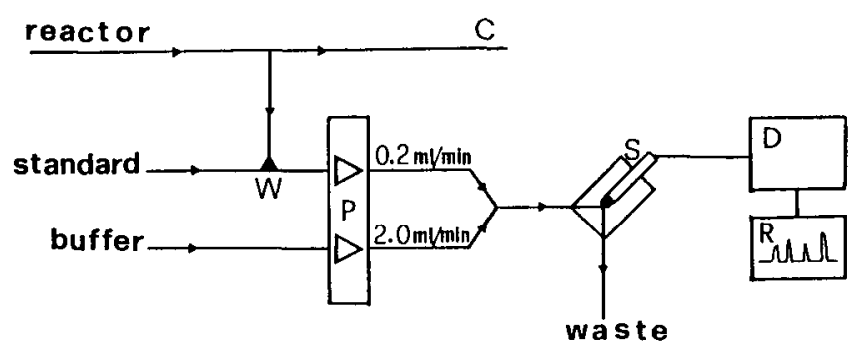

Fig. 3. Diagram of the in-line glucose determination: $P$, peristaltic pump; $W$, three-way stopcock; $\mathrm{S}$, sensor; $\mathrm{D}$, detector; $\mathrm{R}$, recorder.

action was related to the metabolite concentration. The results shown in Fig. 2 are promising for clinical application in combination with the artificial pancreas.

In-line monitoring of glucose produced by an enzymatic reactor

An electrochemical biosensor for the in-line determination of glucose, produced by the enzymatic hydrolysis of cellobiose, was developed. A hydrogen peroxide sensor coupled with glucose oxidase was used. The sensor is based on the well known oxidation of glucose catalyzed by glucose oxidase. The glucose sensor was connected to the system that produced glucose from cellobiose, as shown in Fig 3. The pump aspirated the effluent from the reactor and, after 1:10 dilution with $0.1 \mathrm{~mol} \mathrm{l}^{-1}$ phosphate buffer $(\mathrm{pH} \mathrm{6.0)}$ ), passed the sample to the flow cell where the glucose sensor was fixed. The three-way stopcock provided the standard solutions for calibrating the sensor. The reactor was fed with a buffer solution (citrate $25 \mathrm{mmol}^{-1}, \mathrm{pH} 4.4$ ) containing cellobiose (5- 

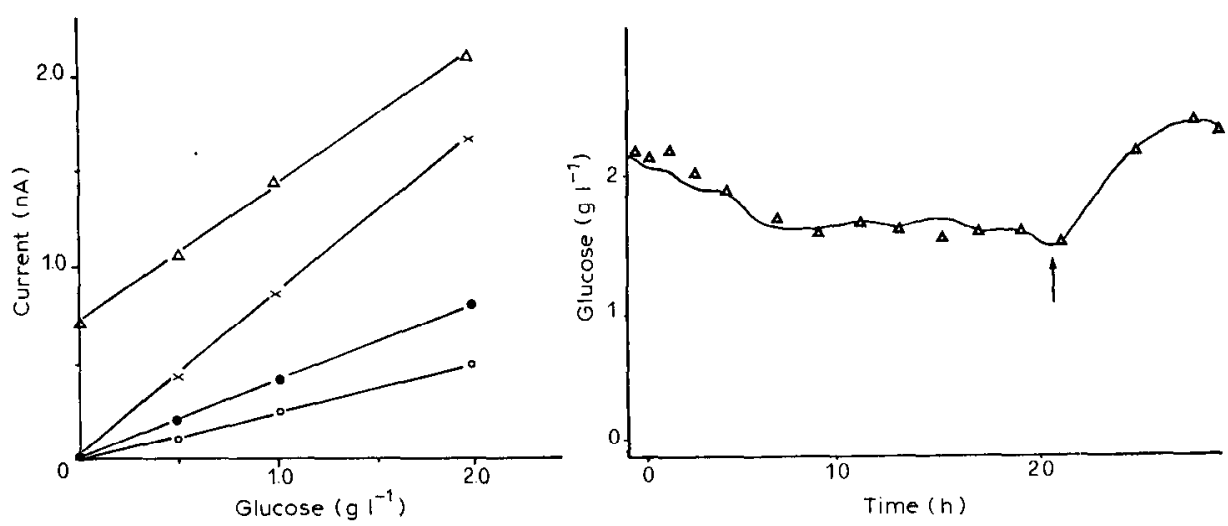

Fig. 4. Calibration curves for glucose with different enzyme preparations of glucose oxidase: $(\triangle)$ Type VII immobilized on Pall BIA membrane, glucose standards containing $10 \mathrm{~g}^{-1}$ cellobiose; (X) Type VII immobilized on Pall BIA membrane, glucose standards without cellobiose; (O) Type V purified and immobilized with the BSA procedure, glucose standards containing $10 \mathrm{~g} \mathrm{l}^{-1}$ cellobiose; (O) Type V purified and immobilized on Pall BIA membrane, glucose standards containing $10 \mathrm{~g} \mathrm{l}^{-1}$ cellobiose.

Fig. 5. Comparison between the in-line determination of glucose (solid line), and a reference method applied to discrete samples taken from the effluent. At the time marked with an arrow, the glucose concentration increased because a more concentrated solution of cellobiose was fed into the bioreactor.

$20 \mathrm{~g} \mathrm{l}^{-1}$ ). The calibration procedure, with the feed solution to which standard amounts of glucose were added, was repeated every two hours.

The assembly of this glucose sensor, which is useful for aqueous solutions with high concentration of cellobiose, required a purified enzyme because commercial preparations of glucose oxidase contain impurities like amylase, maltase, invertase and galactose oxidase which react with cellobiose to give spurious and sluggish signals. When a glucose sensor was assembled with a purified preparation (Type V), better results were obtained. Calibration curves obtained for glucose with sensors based on different enzyme preparations immobilized with two procedures are shown in Fig. 4. The purified enzyme is less active, but the corresponding sensor is more selective and cellobiose does not interfere. The immobilization procedure involving bovine serum albumin (BSA) was chosen because higher current and faster response were observed. The results obtained with the continuous system were compared with a reference method (Fig 5); the correlation was satisfactory $(Y=0.1+0.9 X$, $\left.r^{2}=0.967\right)$.

\section{Biosensors in milk analysis}

Lactate determination. A flow system (flow rate $0.2 \mathrm{ml} \mathrm{min}^{-1}$ ) based on the same lactate sensor as described above, placed in a flow cell with an estimated 
dead volume of $40 \mu \mathrm{l}$, was used in milk analysis. Solutions were aspirated by a peristaltic pump through the cell. Serial dilutions of lactate standard were pumped and the currents generated were recorded to produce a calibration curve; samples were diluted 1:200 before introduction. Phosphate buffer $(\mathrm{pH}$ $7.0,0.1 \mathrm{~mol} \mathrm{l}^{-1}$ ) was used to prepare all standards and milk solutions. Typical outputs are shown in Fig. 6.

To ascertain the suitability of this amperometric method, a spectrophotometric reference method was used for comparison. Evaluation of the two sets of results by linear least-squares showed good correlation $\left(r^{2}-0.997\right)$. The advantage of the present method is that it is faster than the reference procedure, as it requires no sample pretreatment other than dilution, whereas the reference method needs protein-free samples (i.e., pre-treatment with perchloric acid and centrifugation) and incubation for $30 \mathrm{~min}$ to complete the enzymatic reaction.

As stated earlier, the lactate concentration in milk samples increases because of bacterial action producing lactic acid. Figure 7 shows the effect of ageing of a fresh milk sample on its $\mathrm{pH}$ and lactate concentration; the sample was stored in a thermostat $\left(37^{\circ} \mathrm{C}\right)$ and lactate and $\mathrm{pH}$ were measured at certain times. The curves shown are typical of bacterial growth (lag and log stages). At the time marked STIR, when a steady state was reached, the solution was stirred vigorously to provide more oxygen; lactate production resumed as a result of bacterial growth, for which oxygen is a limiting factor. At the time marked ADJ where the $\mathrm{pH}$ was adjusted to 7.03 with sodium hydroxide, a similar effect was noted again. This last experiment shows the wide applicability of this bioelectrochemical sensor in various areas of milk technology. Its fast and precise response allows its use in milk quality control and also in monitoring particular processes for milk products such as cheese and yoghurt.

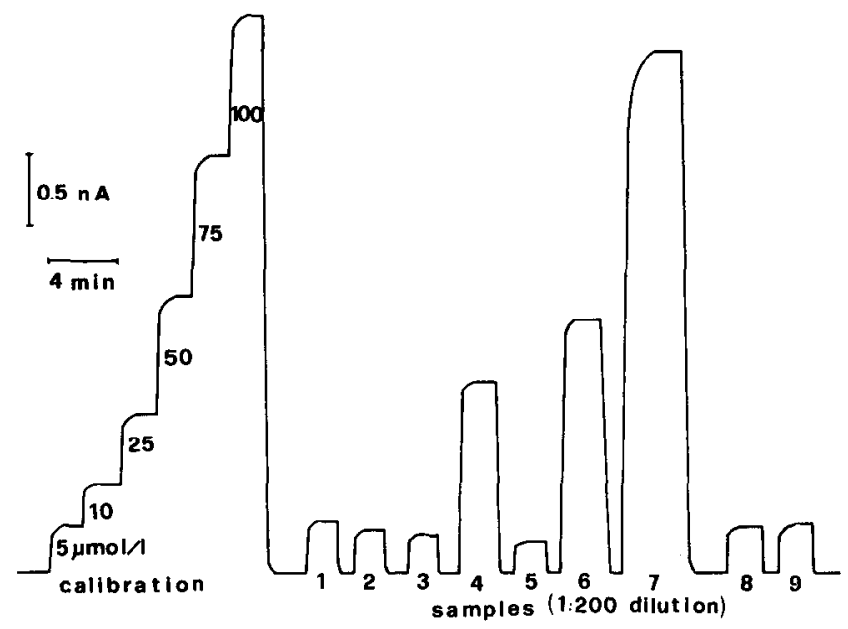

Fig. 6. Lactate determination: current recorded with standard solutions and milk samples diluted $1: 200$. 


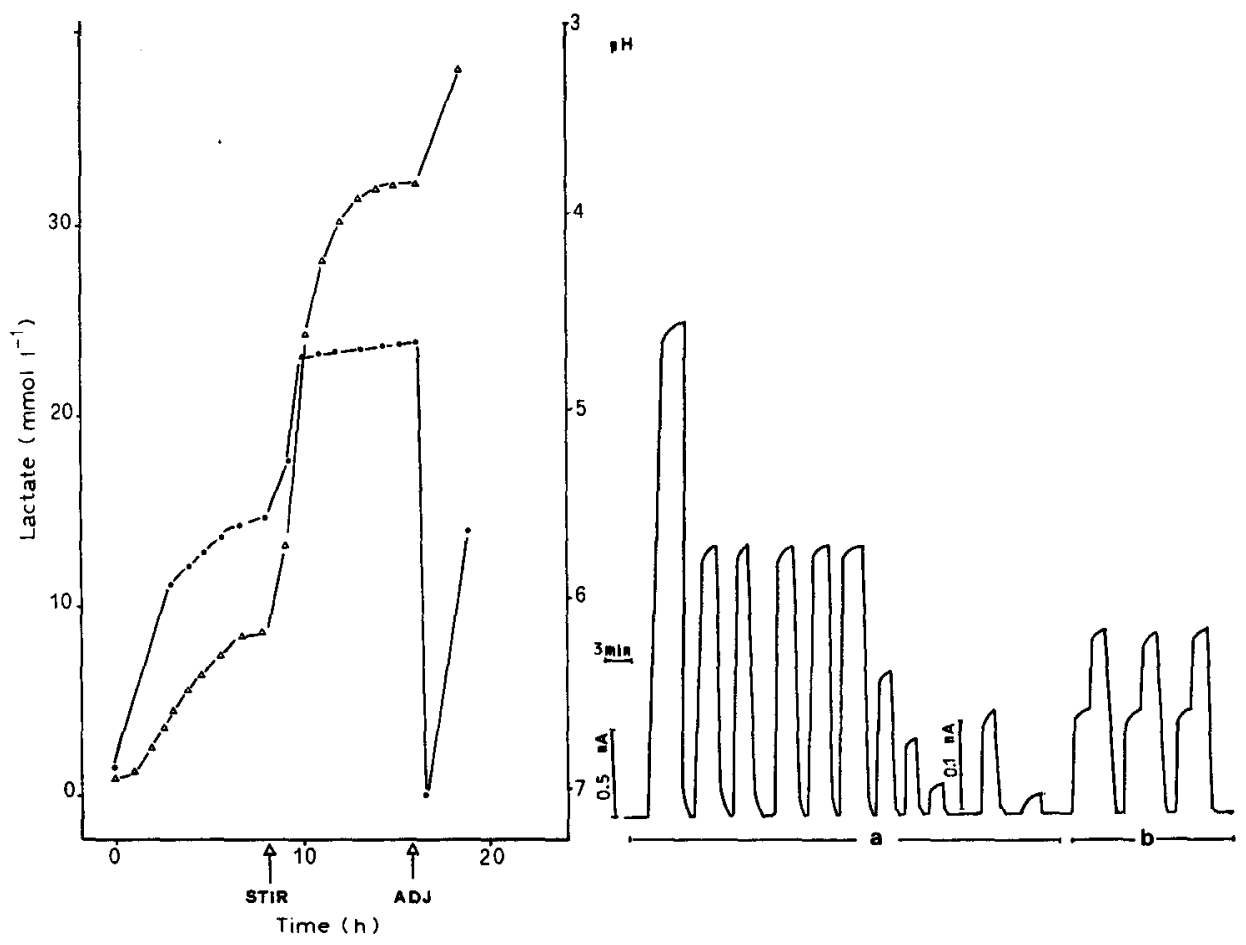

Fig. 7. Variation of lactate $(\Delta)$ and $\mathrm{pH}(O)$ in a fresh milk sample stored at $37^{\circ} \mathrm{C}$ as a function of time. At the arrow STIR, the sample was stirred to enhance oxygen availability; at the arrow ADJ, pH was adjusted to 7.03 .

Fig. 8. (a) Recordings for standard solutions: a $4 \%(w / v)$ lactose solution was diluted (from left to right) 1:50, 1:100, 1:200, 1:400, 1:800, 1:2000, 1:10 000. (b) Example of the assay procedure, based on comparison of the current obtained by injection of sample (diluted 1:100) and of standard added to sample.

Lactose determination. Two different procedures for the determination of lactose based on an enzymatic electrochemical sensor were also examined. They differ only in the enzymes coupled to the electrochemical sensor.

In the first method, both enzymes were immobilized on the electrode surface: glucose oxidase with bovine serum albumin (BSA) and $\beta$-galactosidase with polyazetidine, two dialysis membranes serving as the support. Then, the two membranes were fixed on the platinum electrode with an 0 -ring. The procedure is simple and fast. A milk sample $(100 \mu \mathrm{l})$ was added to $10 \mathrm{ml}$ of phosphate

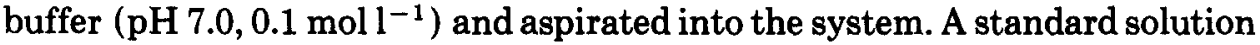
of lactose $(50 \mu \mathrm{l}, 4 \% \mathrm{w} / \mathrm{v})$ in the same buffer was added to a 5 -ml portion of this solution. The current produced by the lactose was recorded for both solutions (Fig. 8). The time required for each sample was short (4 min for each determination, with $95 \%$ of the response in $40 \mathrm{~s}$ ) and the reproducibility was 


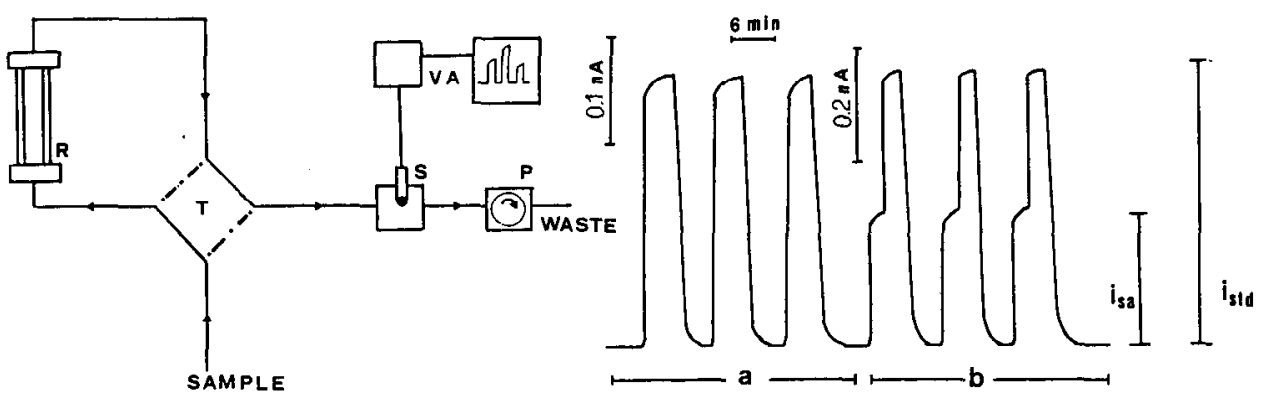

Fig. 9. Schematic diagram of the manifold used: $R, \beta$-galactosidase rector; $P$, peristaltic pump; $T$, four-way valve; $S$, sensor; VA, voltammeter and recorder. The reactor was included only in the second procedure for lactose determination.

Fig. 10. Recordings obtained with the flow system including the $\beta$-galactosidase reactor: (a) standard 4\% (w/v) lactose solution diluted 1:100; (b) sample of fresh milk and sample plus standard addition.

within $1 \%$. These results are better than those previously published [10]. This improved behaviour is due to the procedures used for enzyme immobilization, i.e., the enzymes are immobilized separately and then brought together in the final assembly. When the immobilization reactions proceed on the same membrane, glucose oxidase competes with $\beta$-galactosidase and the result is that the first enzyme is bound more quickly than the second.

The second method for the determination of lactose is based on a flow system in which the two enzymes are used separately; $\beta$-galactosidase is covalently immobilized by the polyazetidine procedure in a reactor with hollow fibers and glucose oxidase is immobilized on the sensor surface by the BSA procedure. The general assembly of the system with a $\beta$-galactosidase reactor is shown in Fig. 9. This procedure was used for samples (1:100 dilution) and a typical recording is shown in Fig. 10. With this manifold, glucose could be determined in milk samples by switching valve T (Fig. 9) to prevent passage through the $\beta$-galactosidase reactor. This procedure was applied for the determination of glucose in "lactose-free" milk.

The varous examples reported above illustrate the wide applicability of electrochemical biosensors. The main features of these sensors, i.e., their short response times, sensitivity, selectivity and reproducibility, emphasize their value for solving analytical problems in biotechnology, medicine, and food industry.

\section{REFERENCES}

1 E.J. Fogt, L.M. Dodd and A.H. Clemens, Clin. Chem. (N.Y.), 24 (1978) 1366.

2 D. Guisard, J.P. Gonand and G. Debry, Clin. Chim. Acta, 36 (1972) 281. 
3 2nd Int. Symp. Advanced Models for the Therapy of Insulin Dependent Therapy, Assisi, Italy, April, 1986, Session IV.

4 M. Mascini, F. Mazzei, D. Moscone, G. Calabresi and M. Massi-Benedetti, Clin. Chem. (N.Y.), 33 (1987) 591.

5 M. Mascini, S. Fortunati, D. Moscone, G. Palleschi, M. Massi-Benedetti and P. Fabietti. Clin. Chem. (N.Y.), 31 (1985) 451.

6 S. Wald, C.R. Wilke and H.W. Blanch, Biotechnol. Bioeng., 26 (1984) 221.

7 A. Adami, C. Fabiani, M. Leonardi and M. Pizzichini, in X. Drioli and M. Nakagaki (Eds.), Membranes and Membrane Processes, Plenum, New York, 1986, p. 241.

8 C. Fabiani, G. Giubileo, M. Pizzichini and V. Violante, Biotechnol. Bioeng., 28 (1986) 000.

9 P.F.A. Turner and J.C. Pickup, Biosensors, 1 (1985) 1.

10 R. Pilloton, M. Mascini, I.G. Casella, M.R. Festa and E. Bottari, Anal. Lett., 20 (1980) 87.

11 M. Mascini, M. Iannello and G. Palleschi, Anal. Chim. Acta, 146 (1983) 135.

12 M. Mascini and F. Mazzei, Anal. Chim. Acta, 192 (1987) 9.

13 L.L. Wood and G.J. Calton, Biotechnol. Discuss., 2 (1984) 1081. 